\title{
Clinical and Economic Evaluation of Repository Corticotropin Injection: A Narrative Literature Review of Treatment Efficacy and Healthcare Resource Utilization for Seven Key Indications
}

\author{
Michael Philbin · John Niewoehner - George J. Wan
}

Received: May 1, 2017 / Published online: June 28, 2017

(C) The Author(s) 2017. This article is an open access publication

\begin{abstract}
Introduction: Repository corticotropin injection (RCI; H.P. Acthar ${ }^{\circledR}$ Gel; Mallinckrodt Pharmaceuticals Inc., Hampton, NJ) is a highly purified, prolonged-release porcine preparation of adrenocorticotropic hormone (ACTH) analogue that is FDA-approved for treatment of 19 autoimmune and inflammatory disorders. The diverse physiological actions of RCI at the melanocortin receptors (MCRs) affect processes involved in inflammation, pigmentation, steroidogenesis, and immunomodulation. Although RCI has been approved to treat inflammatory and autoimmune diseases for more than 60 years, recent progress in understanding both MCRs and the effects of RCI in modulating immune responses has led to increased interest in RCI as a therapeutic choice. The objective of this narrative literature review is to summarize key clinical and economic data
\end{abstract}

Enhanced content To view enhanced content for this article go to http://www.medengine.com/Redeem/ 2898F0604C7E5A8D.

Electronic supplementary material The online version of this article (doi:10.1007/s12325-017-0569-9) contains supplementary material, which is available to authorized users.

M. Philbin · J. Niewoehner · G. J. Wan ( $\square)$

Mallinckrodt Pharmaceuticals Inc., Hampton, NJ, USA

e-mail: george.wan@mallinckrodt.com on RCI treatment of seven disorders: infantile spasms (IS), multiple sclerosis (MS) relapses, proteinuria in nephrotic syndrome, rheumatoid arthritis (RA), dermatomyositis/polymyositis (DM/PM), systemic lupus erythematosus (SLE), and symptomatic sarcoidosis based on published literature and product information. An extended report is available as the Academy of Managed Care Pharmacy (AMCP) Formulary dossier for H.P. Acthar ${ }^{\circledR}$ Gel.

Methods: Key studies of clinical efficacy and healthcare utilization and cost from 1956 to 2016 are summarized.

Results: The evidence supports the efficacy of $\mathrm{RCI}$ across the seven indications. RCI is effective as a first-line therapy for IS. For the other six conditions, RCI may improve clinical outcomes during exacerbations or when the condition is resistant to conventional treatments. Use of RCI is associated with reduced use of biologics, corticosteroids, and disease-modifying antirheumatic drugs. Initiation of RCI therapy in patients with IS, MS, RA, SLE, or DM/PM has been associated with lower post-therapy healthcare utilization and medical costs, including decreases in hospitalizations, hospital length of stay, outpatient visits, and emergency department visits.

Conclusion: The evidence suggests that RCI may improve inflammatory and autoimmune disease control and patient quality of life, particularly in complex patients, and yield 
healthcare cost savings that demonstrate the medicine's value.

Funding: Mallinckrodt Pharmaceuticals Inc.

Keywords: АCTH; H.P. Acthar $^{\circledR}$ Gel; Dermatomyositis; Healthcare utilization; Infantile spasms; Multiple sclerosis relapse; Nephrotic syndrome; Rheumatoid arthritis; Sarcoidosis; Systemic lupus erythematosus

\section{INTRODUCTION}

Autoimmune diseases have wide-ranging effects in the body, including neurologic, renal, musculoskeletal, dermatologic, and pulmonary effects, and can lead to dysfunction of multiple organs and tissues. These autoimmune diseases can be associated with substantial patient morbidity, disability, and impaired quality of life and they may impose significant long-term humanistic and economic burdens on families, healthcare systems, and society.

Autoimmune diseases are treated with a variety of therapeutic agents, including corticosteroids, immunosuppressants, immunomodulators, disease-modifying antirheumatic drugs (DMARDs), biologics, and nonsteroidal anti-inflammatory agents. Repository corticotropin injection (RCI; H.P. Acthar ${ }^{\circledR}$ Gel; Mallinckrodt Pharmaceuticals Inc., Hampton, NJ, USA) is a highly purified, prolonged-release preparation of adrenocorticotropic hormone (ACTH) in a 16\% gelatin formulation that is administered via intramuscular or subcutaneous injection. It has been in clinical use for more than 60 years. RCI was first approved by the US Food and Drug Administration (FDA) in 1952, before the 1962 enactment of the Kefauver-Harris Amendment to the Federal Food, Drug and Cosmetic Act, an amendment that requires drug manufacturers to provide proof of effectiveness and safety before approval. For this reason, much of the available research on RCI consists of case series, uncontrolled or small randomized clinical trials, and retrospective analyses. Under the FDA's Drug Efficacy Study Implementation (DESI) program that was initiated following the Kefauver-Harris Amendment, RCI has been reviewed three times (in 1977, 1979, and 2010). With the most recent label update in 2015, RCI is currently approved for 19 indications (Table S1) (supplementary table is available online) [1]. RCI is the only naturally sourced form of ACTH approved for use in the USA.

RCI binds to melanocortin receptors (MCRs) and may have a diverse range of actions, including effects on inflammation, pigmentation, steroidogenesis, and immunomodulation $[1,2]$. Current understanding of the melanocortin system points to a set of signaling pathways that play a role in inflammation control and immunomodulation [3]. In recent years, progress in understanding both MCRs and the effects of RCI in modulating immune responses has led to a renewed interest in RCI as a therapeutic choice [2].

The objective of this narrative literature review is to summarize the key clinical and economic data contained in the Academy of Managed Care Pharmacy (AMCP) Formulary dossier for H.P. Acthar ${ }^{\circledR}$ Gel for seven key indications: infantile spasms (IS), multiple sclerosis (MS) relapses, proteinuria in nephrotic syndrome, rheumatoid arthritis (RA), dermatomyositis/polymyositis (DM/PM), systemic lupus erythematosus (SLE), and symptomatic sarcoidosis $[1,4]$.

This article is based on previously conducted studies and does not involve any new studies of human or animal subjects performed by any of the authors.

\section{CLINICAL VALUE OF REPOSITORY CORTICOTROPIN INJECTION}

A number of studies in different therapeutic areas have assessed the clinical efficacy of RCI before and after treatment or compared RCI with placebo or other common therapies. These are described below and summarized in Table 1.

\section{Infantile Spasms}

IS, also known as West syndrome, refers to a unique epilepsy syndrome of early infancy ( $<2$ years of age) in which patients present with a distinct seizure type [5-7]. Untreated IS is 


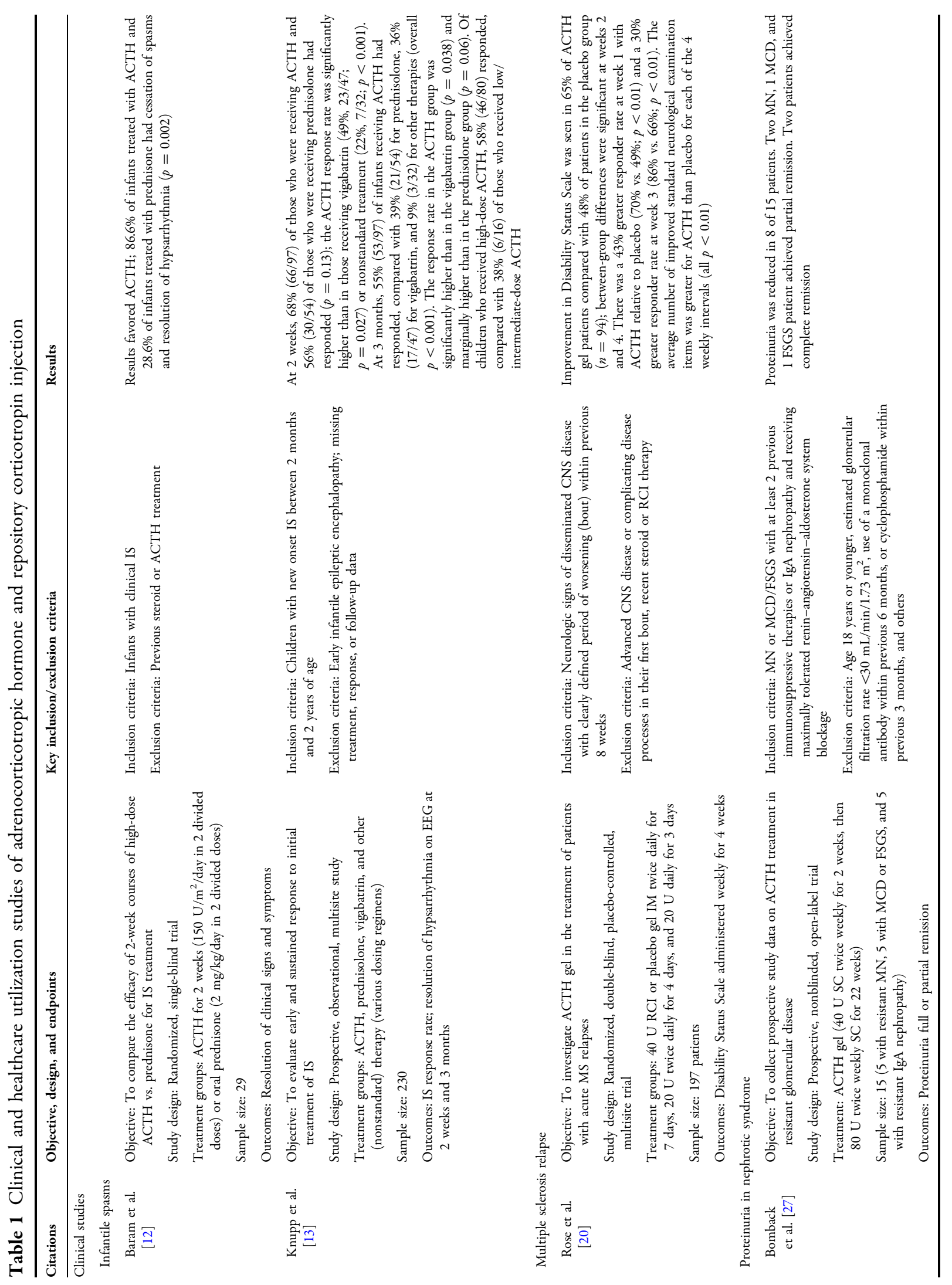




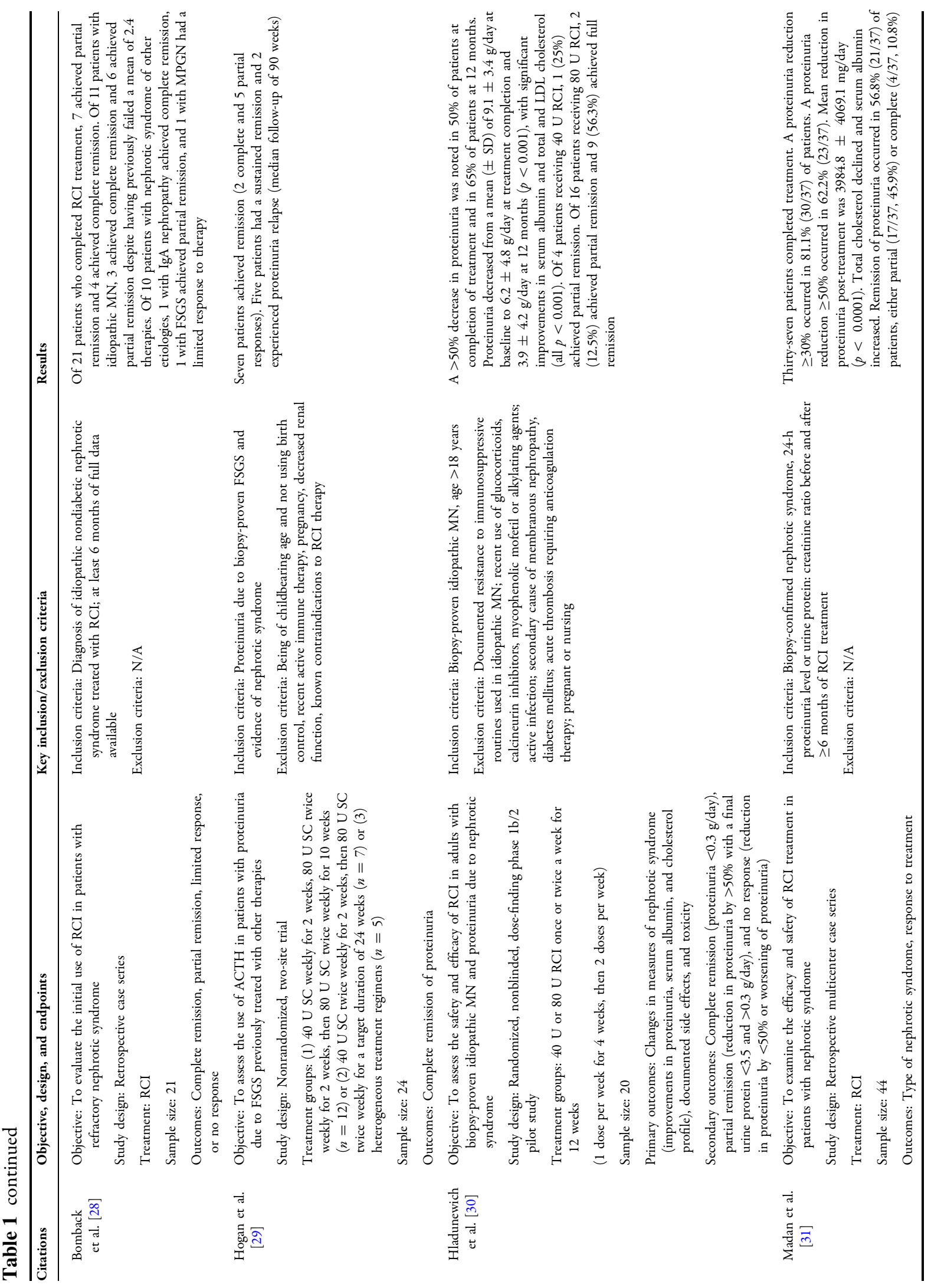




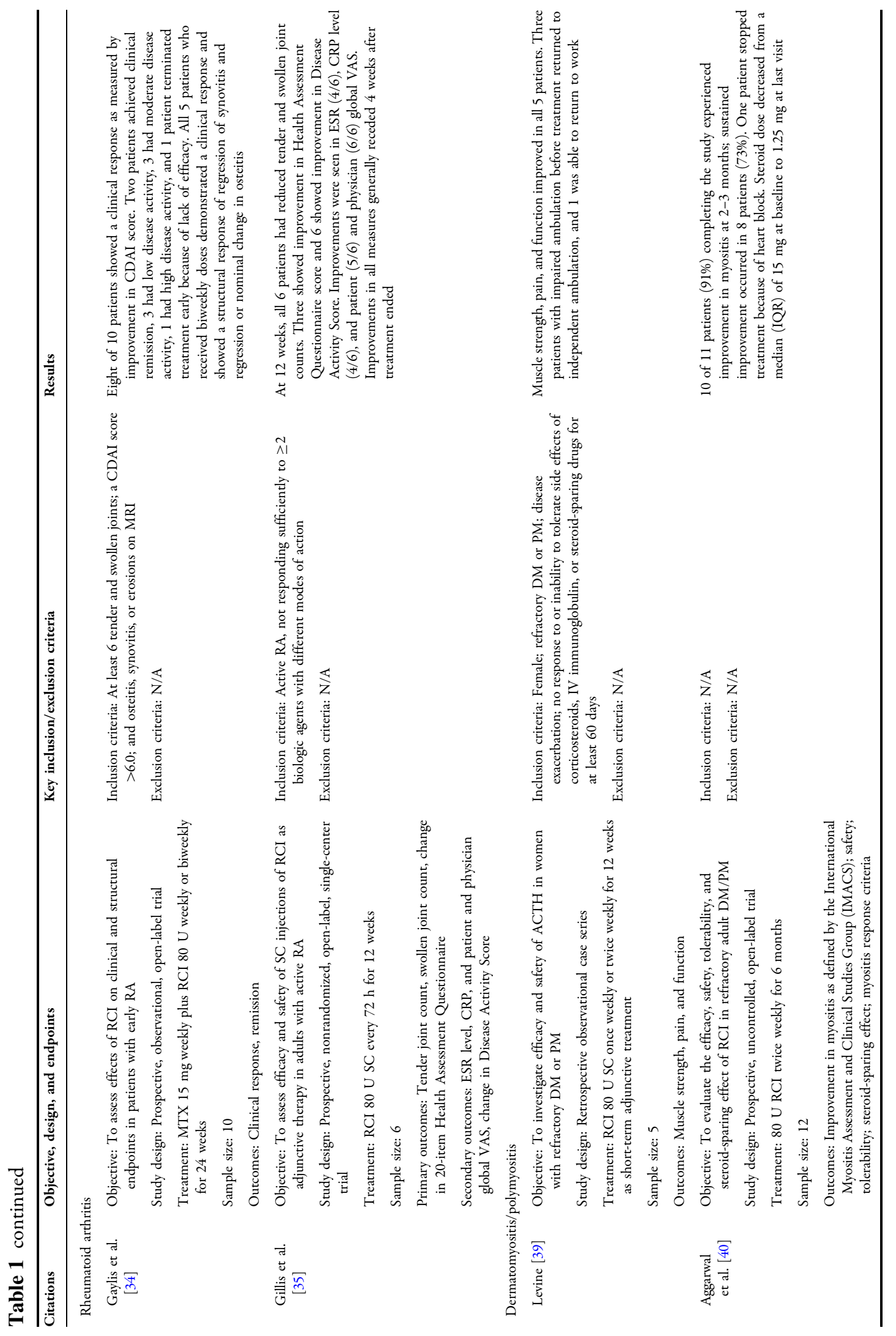




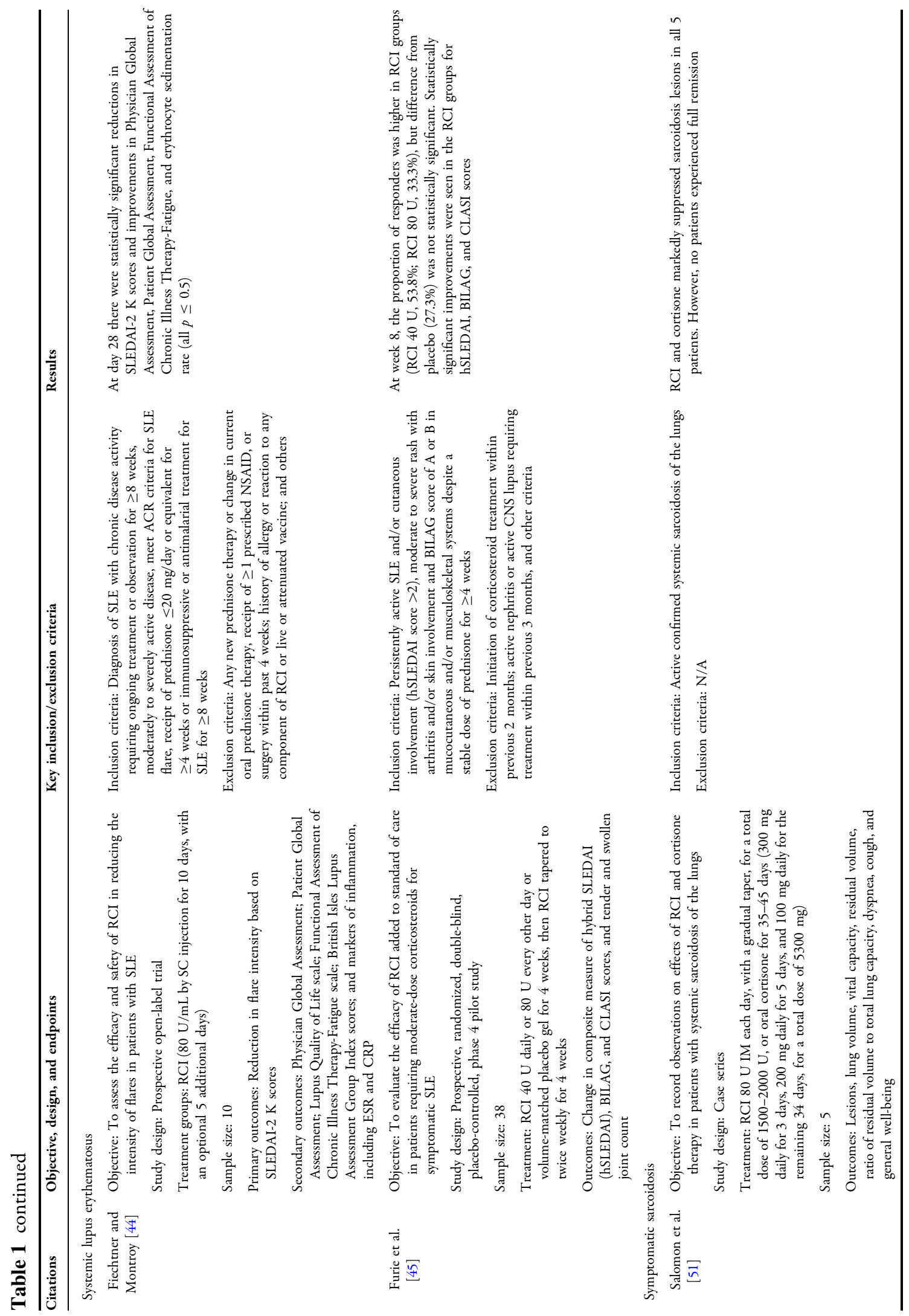




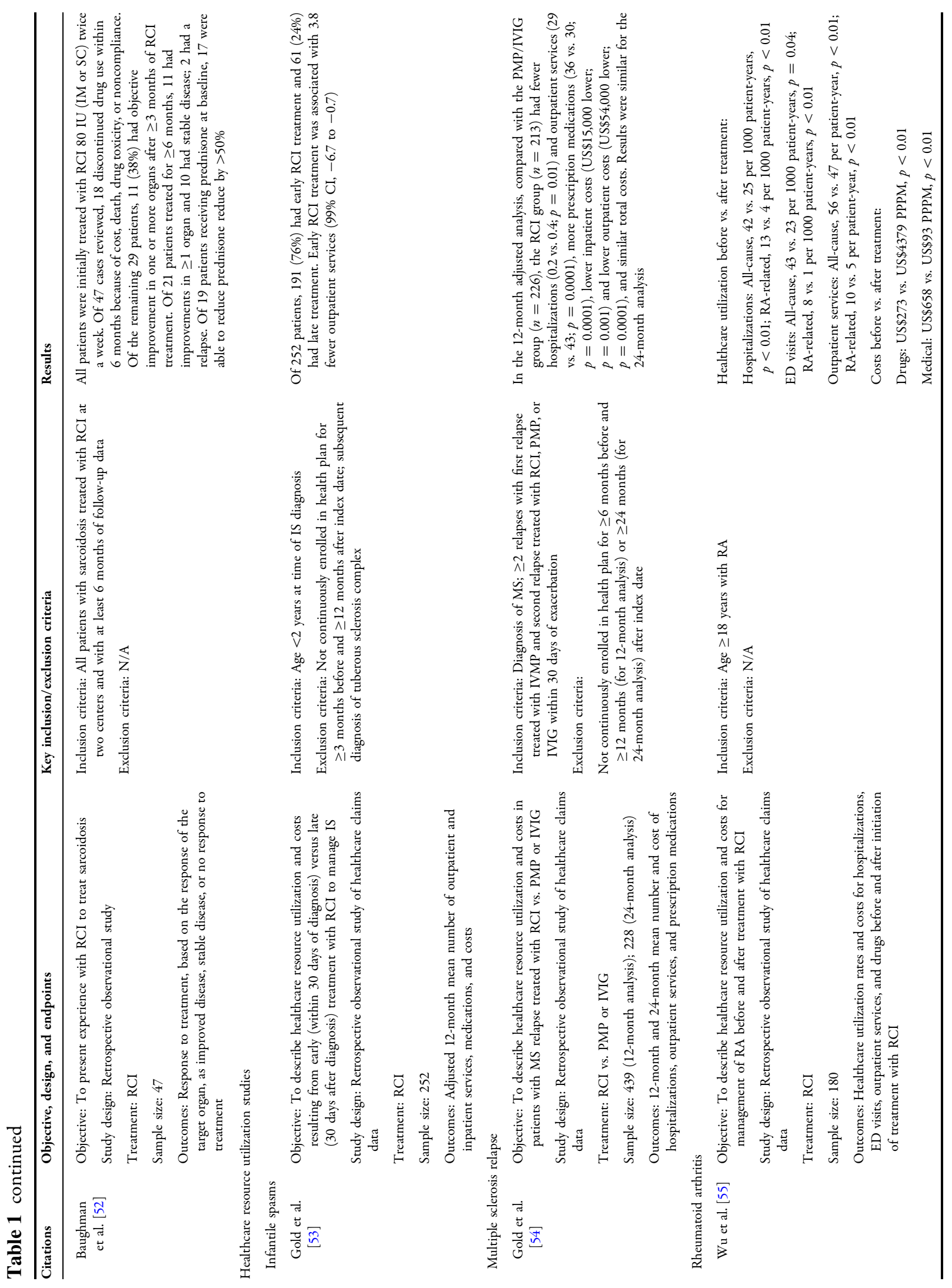




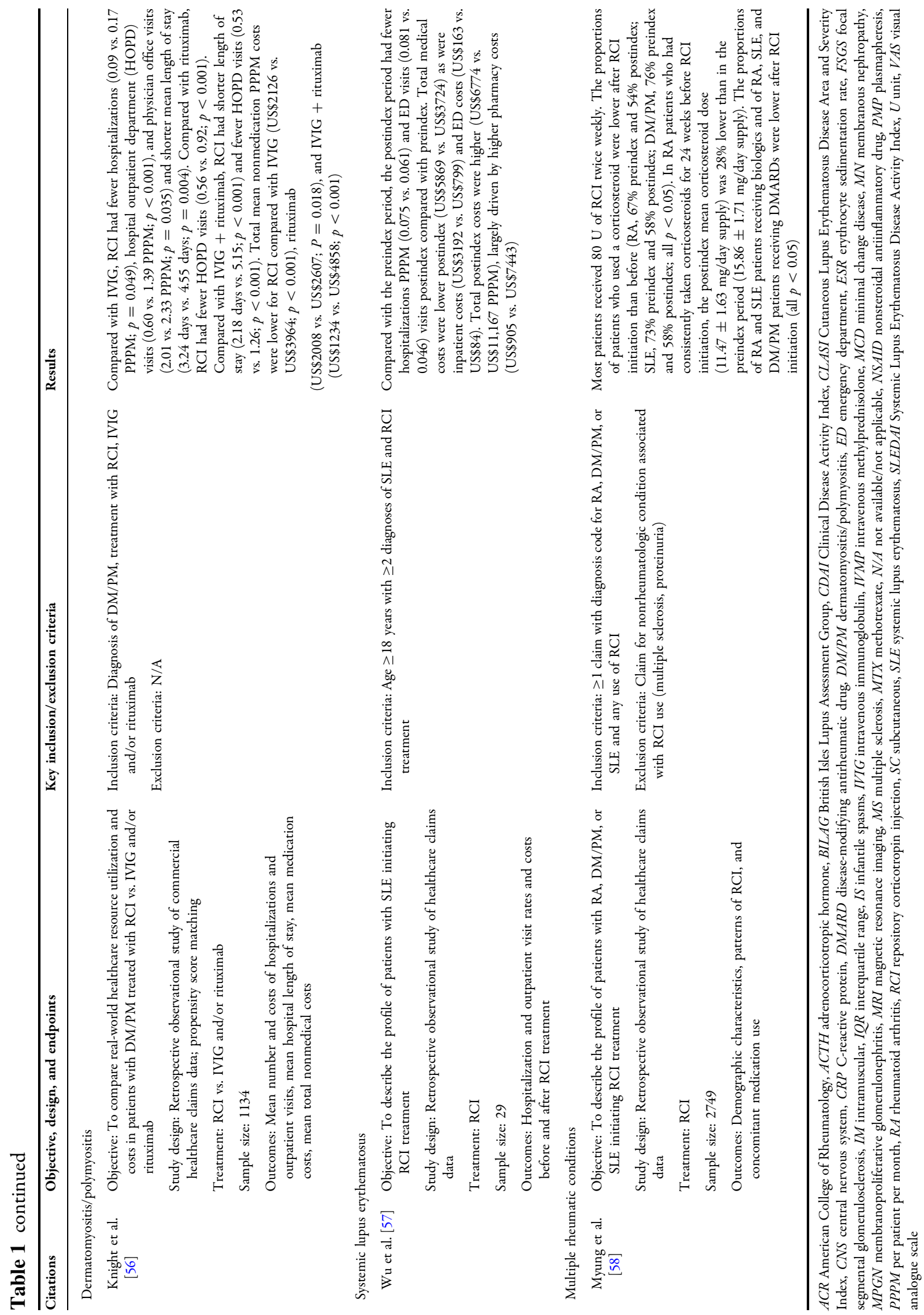


associated with developmental delay, autism, mental retardation, and an $11 \%$ mortality rate by age 2 years [5-8]. In a study of 244 infants aged 0-18 months, a delay in time to diagnosis following initial presentation was associated with cognitive abnormalities (median time to diagnosis, 18 vs. 6 days; $p<0.001$ ) and motor abnormalities (median time to diagnosis, 17.5 vs. 6 days; $p<0.001$ ) [9]. Outcomes may therefore be improved by early recognition of IS and immediate, effective treatment $[6,8,10]$.

Compared with prednisone and conventional antiepileptic drugs, RCI may be more efficacious for the treatment of IS. RCI has orphan drug designation in the USA for treatment of IS [11]. In a US randomized controlled trial of 29 infants (median age, 6 months), $86.6 \%$ of infants treated with RCI (150 U/ $\mathrm{m}^{2} /$ day) and $28.6 \%$ of infants treated with prednisone $(2 \mathrm{mg} / \mathrm{kg} / \mathrm{day})(p=0.002)$ had cessation of spasms and resolution of hypsarrhythmia after 2 weeks of therapy [12]. The most frequently reported adverse events in RCI-treated infants were irritability and voracious appetite.

More recently, a US study of 230 infants at 22 centers participating in the National Infantile Spasms Consortium reported that $55 \%$ of infants receiving $\mathrm{RCI}$ as initial treatment responded to therapy, compared with $39 \%$ for oral corticosteroids, $36 \%$ for vigabatrin, and $9 \%$ for other therapies $(p<0.001)$ [13].

The 2004 IS guideline of the American Academy of Neurology and Child Neurology Society recommends ACTH for short-term control of IS, supported by strong (level B) evidence [5]. Although the guideline refers to ACTH, RCI is the only form of ACTH approved for use in the USA, as noted previously. Based on three studies of RCI [14-16] and one study of synthetic ACTH [14-16], the 2012 guideline update recommends that low-dose ACTH be considered as an alternative to high-dose ACTH for treatment of IS (level B evidence) [6].

\section{Multiple Sclerosis Relapses}

MS is a complex and chronic demyelinating autoimmune neurological disorder. It is most frequently diagnosed between 20 and 40 years of age and is a leading cause of disability in this age group [17]. Acute relapses in MS may present with severe symptoms. To decrease inflammation and hasten recovery, a short course of corticosteroids (high-dose intravenous [IV] or oral) is often prescribed. However, if a patient does not respond or presents with breakthrough disease, ACTH, IV immunoglobulin (IVIG), or plasma exchange (plasmapheresis; PMP) may be required [18]. In the USA, RCI was first approved for treatment of MS exacerbations in 1978 and is currently indicated for treatment of acute exacerbations of MS in adults $[1,19]$. Intravenous immunoglobulin and PMP are not FDA-approved therapies for relapses in MS.

The efficacy of RCI compared with placebo or other common therapies for the treatment of MS relapses has been evaluated. A large randomized, controlled, double-blind, multicenter trial found that RCI was superior to placebo for reducing the duration of relapse [20]. In a small study comparing routes of administration, intramuscular and subcutaneous administration of a 5-day course of RCI produced a comparable decrease in symptoms [21].

Both the National Multiple Sclerosis Society and the American Academy of Neurology have noted that alternative treatment options are needed for patients who experience MS relapse but do not respond to, cannot tolerate, or do not wish to take steroids [22, 23]. Thus, for patients needing an alternative treatment, RCI may provide an option.

\section{Proteinuria in Nephrotic Syndrome}

Nephrotic syndrome is a constellation of renal and extrarenal signs and symptoms that includes edema, proteinuria, hypoalbuminemia, lipiduria, hyperlipidemia, and hypercoagulability. It is caused by several systemic diseases and by primary insult to the kidney. Primary renal causes of nephrotic syndrome include minimal change disease, focal segmental glomerulosclerosis, membranous nephropathy, immunoglobulin A (IgA) nephropathy, and membranoproliferative glomerulonephritis, 
and nephrotic syndrome may occur secondary to systemic diseases such as diabetes and systemic amyloidosis [24-26]. In patients with resistant glomerular disease, RCI is a treatment option, along with conventional therapy. It is indicated for inducing diuresis or remission of proteinuria in nephrotic syndrome without uremia of the idiopathic type and in nephrotic syndrome due to lupus erythematosus [1].

Several studies have shown that RCI reduces proteinuria in several subtypes of treatment-resistant nephrotic syndrome: idiopathic membranous nephropathy, focal segmental glomerulosclerosis, minimal change disease, membranoproliferative glomerulonephritis, lupus nephritis, and IgA nephropathy [27-31].

\section{Rheumatoid Arthritis}

RA is an autoimmune disease that leads to progressive destruction of the joints and associated cartilage [32]. It is associated with several comorbidities, including cardiovascular and pulmonary manifestations, skeletal disorders, cognitive effects, infection, and malignancies [33].

RCI is an FDA-approved adjunctive therapy for short-term administration (to tide the patient over an acute episode or exacerbation) and for use in "selected cases who may require low-dose maintenance therapy" [1]. It has been shown to be effective in combination with methotrexate in terms of clinical response, inducing remission and slowing structural changes, with few serious adverse events observed [34, 35]. In an open-label study, 10 patients with early RA and at least six tender and swollen joints were treated with methotrexate plus RCI either weekly $(n=5)$ or biweekly $(n=5)$ [34]. An improved Clinical Disease Activity Index (CDAI) score was observed in eight patients. In the five patients who received RCI biweekly, both clinical and structural improvements were seen. A second open-label study of RA patients with an inadequate response to two or more biologic agents with different modes of action $(N=6)$ found that treatment with $\mathrm{RCI}$ for 12 weeks led to reductions in tender and swollen joint counts and improvements in Disease Activity Score for RA (DAS28), Patient Global Visual Analogue Scale (VAS), and Physician Global VAS in all patients [35].

\section{Dermatomyositis and Polymyositis}

$\mathrm{DM}$ and $\mathrm{PM}$ are autoimmune connective tissue diseases that are characterized by chronic inflammation of proximal skeletal muscles. DM also affects the skin and may affect the joints, esophagus, heart, and lungs. Patients with DM or PM typically present with slowly progressive weakness and reduced endurance in the muscles of the neck, shoulders, and pelvis [36-38].

Patients with DM or PM may experience clinical benefit from RCI therapy. In a case series study, five patients experiencing a disease exacerbation who had either failed or experienced adverse effects with conventional treatment were treated with RCI once or twice weekly for 12 weeks [39]. All patients showed improvement in manual muscle testing scores at 12 weeks, and no patients experienced significant adverse events. In an open-label uncontrolled study of RCI (80 U twice weekly for 6 months), 10 of 11 patients (91\%) experienced improvement in myositis as defined by the International Myositis Assessment and Clinical Studies Group (IMACS) [40]. In addition, RCI was found to be generally safe, well tolerated, and steroid sparing.

Other than corticosteroids, RCI is the only FDA-approved drug for the treatment of DM/ PM. It is indicated for use during an exacerbation or as maintenance therapy in selected cases [1].

\section{Systemic Lupus Erythematosus}

SLE is a systemic illness characterized by immune hyperactivity and the production of antinuclear, anticytoplasmic, and antiphospholipid antibodies. Patients typically present with fatigue, malaise, fever, arthralgia, myalgia, headache, loss of appetite, weight loss, and rash. The disease may progress to multiple end-organ involvement, resulting in photosensitivity, arthritis, and serositis as well as renal, 
neurological, and other disorders [41-43]. During the course of the disease, intermittent acute relapses or flares may result in tissue damage. $\mathrm{RCI}$ is indicated during an exacerbation or as maintenance therapy in selected cases of SLE [1].

In a single-arm, uncontrolled, open-label study of 10 female patients with moderate-to-severe SLE who were receiving conventional therapy and experiencing a disease flare, adjunctive RCI treatment for 10 days, with an optional five additional days, led to reduction in disease activity and to improvement in functional status, quality of life, and erythrocyte sedimentation rate at 28 days [44]. No treatment-related serious or unexpected adverse events were observed. In an 8-week randomized controlled trial of RCI for the treatment of persistently active SLE, 38 participants received RCI $80 \mathrm{U}$ every other day $(n=13), \mathrm{RCI} 40 \mathrm{U}$ daily $(n=13)$, or placebo $(n=12)$ [45]. Disease activity was reduced in the RCI groups compared with placebo, including statistically significant decreases in the Hybrid SLE Disease Activity Index and the British Isles Lupus Assessment Group index in both treatment groups at 8 weeks and in tender swollen joint count in the $80 \mathrm{U}$ RCI group at 8 weeks.

\section{Symptomatic Sarcoidosis}

Sarcoidosis is a chronic inflammatory granulomatous disease that primarily affects the lungs (90\% of affected patients have pulmonary involvement), although other organs such as the skin and eyes may be involved [46-48]. The disease follows a variable natural course, ranging from asymptomatic to a progressive disease that may be life threatening [48, 49]. Patients may be asymptomatic or have signs and symptoms such as cough, fever, weight loss, chest pain, painful ankle swelling, painful red nodules on the shins, eye pain, and blurred vision [50]. Treatment is generally reserved for symptomatic disease. RCI is an FDA-approved treatment for symptomatic sarcoidosis [1].

RCI was reported to be effective for symptomatic sarcoidosis as early as the 1950 s. A case series study of patients receiving RCI $(n=4)$ or cortisone $(n=1)$ reported that treatment with RCI led to improvements in lung function and regression of skin, peripheral lymph node, parotid gland, and eye lesions [51]. In a recent retrospective chart review of patients with advanced sarcoidosis, 27 of 29 patients who received RCI (alone or in combination with steroids) for 6 months or longer experienced an improvement in their disease and 11 experienced objective improvement in the health of one or more affected organs. In addition, 17 of 19 patients receiving concomitant prednisone were able to reduce their prednisone dose by more than 50\% [52]. Further evaluation of RCI therapy for symptomatic sarcoidosis is warranted, and prospective studies are underway.

\section{HEALTHCARE UTILIZATION AND COSTS}

Evidence in multiple therapeutic areas suggests that use of RCI decreases healthcare utilization, which may be associated with lower expenditures from a healthcare systems perspective. Key outcomes data are summarized in Table 1 and additional data are available in the AMCP dossier [4]. In some instances, these reductions in resource use may mitigate or potentially negate the increased medication costs through medical cost offsets.

\section{Infantile Spasms}

A claims-based analysis of infants with IS who were treated with RCI demonstrated a resource use benefit associated with early treatment (within 30 days of diagnosis) compared with later treatment ( $\geq 30$ days after diagnosis) [53]. In particular, early use of RCI led to 3.8 fewer outpatient visits $(99 \%$ confidence interval [CI] -6.7 to $-0.7 ; p=0.002$ ) and 4.2 fewer visits for all health services combined (99\% CI -7.9 to $-0.4 ; p=0.005)$.

\section{Multiple Sclerosis Relapses}

An economic analysis of patients with MS relapses who had received prior treatment with 
IV methylprednisone demonstrated decreased healthcare utilization for RCI therapy compared with the two therapies that are used most often for the treatment of MS relapse, IVIG and PMP [54]. Over a 12-month period, the RCI group had 0.2 fewer hospitalizations $(95 \% \mathrm{CI}-0.3$ to $-0.1 ; p=0.01), 15$ fewer outpatient services (95\% CI -20 to $-10 ; p<0.0001$ ), 3.7 fewer days in hospital (95\% CI -4.8 to $-1.1 ; p=0.01), 0.19$ fewer rehabilitation and long-term care facilities services (95\% CI -0.26 to $-0.12 ; p<0.001)$, and 3.2 fewer overall healthcare services $(95 \% \mathrm{CI}$ -5.2 to $-1.1 ; p=0.003$ ) while incurring similar total healthcare costs (US\$106,400 vs. US $\$ 109,400 ; p=0.74)$. Favorable cost outcomes for RCI compared with IVIG or PMP also were observed in a 24-month analysis (inpatient costs US $\$ 17,400$ lower, $p=0.03$; outpatient costs US $\$ 121,000$ lower, $p<0.0001$; total costs US $\$ 33,400$ lower, $p=0.13$ ). Although drug costs for RCI treatment were greater than for IVIG or PMP, they were offset by decreases in inpatient and outpatient costs (93\% medication cost offset at 12 months; $132 \%$ offset at 24 months).

\section{Rheumatoid Arthritis}

In patients with RA, treatment with RCI may result in reduced healthcare utilization and decreased use of other RA medications. The HealthCore Integrated Research Database, a large healthcare claims database containing data for 36.8 million commercial health plan members, was used to retrospectively examine healthcare utilization and costs in patients with RA before and after initiating treatment with RCI [55]. Of 6190 eligible RA patients, 180 patients had received RCI. Treatment with RCI was associated with substantial reductions in the use of corticosteroids, biologics, and nonbiologic DMARDs. The RCI cohort also had lower rates of hospitalization after treatment initiation compared with before (all-cause hospitalizations: 42 preinitiation vs. 25 postinitiation per 1000 patient-years, $p<0.01$; RA-related hospitalizations: 13 vs. 4 per 1000 patientyears, $p<0.01$ ), emergency department visits (all-cause visits: 43 vs. 23 per 1000 patient-years, $p=0.04$; RA-related visits: 8 vs. 1 per 1000 patient-years, $p<0.01)$, and outpatient services (all-cause encounters: 56 vs. 47 per patient-year, $p<0.01$; RA-related encounters: 10 vs. 5 per patient-year, $p<0.01)$. Patients incurred higher RA-related drug costs after RCI initiation compared with before initiation (US\$4379 vs. US\$273 per patient per month [PPPM], $p<0.01)$. However, RA-related medical costs were substantially lower after RCI initiation than before (US\$93 vs. US\$658 PPPM, $p<0.01$ ) because of fewer inpatient, outpatient, and emergency department services. The reduction in medical costs offset RCI drug costs by 14-30\%.

\section{Dermatomyositis and Polymyositis}

An economic analysis of 2009-2014 US claims data for patients with DM/PM found a decrease in healthcare utilization in patients treated with RCI [56]. Patients treated with RCI $(n=132)$, IVIG $(n=1150)$, or rituximab $(n=562)$ were propensity score-matched on demographic and clinical characteristics and prior medical resource utilization. The RCI group had fewer mean hospitalizations than the IVIG group (0.09 vs. 0.17 PPPM; $p=0.049)$, shorter mean length of stay (3.24 vs. 4.55 days; $p=0.004$ ), and fewer hospital outpatient department visits (0.60 vs. 1.39 PPPM; $p<0.001)$ and physician office visits (2.01 vs. 2.33 PPPM; $p=0.035$ ), but similar numbers of emergency department visits (0.04 vs. 0.05 PPPM; $p=0.472$ ). RCI patients also had fewer hospital department outpatient visits than patients receiving rituximab or IVIG + rituximab and shorter hospital length of stay. Total mean nonmedication costs were significantly lower in the RCI group than in the IVIG group (US\$2126 vs. US\$3964; $p<0.001$ ), rituximab group (US\$2008 vs. US\$2607; $p=0.018)$, and IVIG + rituximab group (US\$1234 vs. US\$4858; $p<0.001$ ).

\section{Systemic Lupus Erythematosus}

In an analysis of healthcare utilization in patients with SLE, 9944 eligible patients (age $\geq 18$ years) were identified using US commercial 
claims data for 2006-2015 [57]. Of this group, 29 patients had initiated RCI therapy, with the drug initiated an average of 23 months after diagnosis of SLE. Healthcare utilization in the period before RCI initiation (average duration, 23 months) and the period after initiation (average follow-up, 24 months) was compared. Decreases in mean number of hospitalizations and ED visits PPPM were observed postinitiation. Drug costs increased from US\$905 PPPM to US\$7743 PPPM postinitiation; however, SLE-related medical costs decreased from US\$3301 PPPM to US\$893 PPPM $(p=0.02)$, primarily owing to lower hospitalization costs, offsetting approximately one-third of the increase in drug costs.

\section{Multiple Rheumatologic Conditions}

In an analysis of a pooled population of patients with various rheumatologic conditions (RA, $n=1269$; SLE, $n=874$; DM/PM; $n=606$ ), the proportions of patients who used corticosteroid therapy were significantly lower after RCI initiation (reduced from $67 \%$ preindex to $54 \%$ postindex for RA, from $73 \%$ to $58 \%$ for SLE, and from $76 \%$ to $58 \%$ for DM/PM; $p<0.05$ for all comparisons) [58]. Furthermore, the proportions of patients who were receiving biologics and DMARDs were also significantly lowered after initiation of RCI treatment.

General clinical practice is to use RCI as a late-line therapy in patients with autoimmune flares or whose disease has been inadequately controlled with first-line therapies. Taken together, these various retrospective analyses suggest that treatment with RCI is associated with reduced healthcare utilization in patients with moderate to severe disease progression. The lower healthcare utilization may reflect an improvement in disease control, corresponding to the improvements in clinical outcomes observed in the studies summarized above, and warrants additional research.

\section{CONCLUSION}

RCI is used to treat several serious and rare autoimmune conditions. There has been a renewed interest in RCI, partly because of recent progress in understanding the mechanisms by which RCI modulates immune responses. Currently, RCI is considered first-line therapy for IS; for other autoimmune disorders reviewed here it is used primarily as later-line therapy in patients experiencing a disease exacerbation or as adjunctive or maintenance therapy in selected patients who do not respond to or are intolerant of conventional treatment.

More than two dozen clinical trials of RCI in the treatment of MS relapses, symptomatic sarcoidosis, SLE, RA, optic neuritis, proteinuria, $\mathrm{DM}$, and other diseases and conditions are currently registered in the National Institutes of Health clinical trials database (http://www. ClinicalTrials.gov); it is anticipated that this research will greatly improve our understanding of the appropriate use of RCI. The large burden that autoimmune diseases impose on patients, families, healthcare systems, and society warrants continued research to expand the knowledge base of effective therapeutic options for these challenging disorders.

The studies summarized in this review support the potential clinical efficacy of RCI across several autoimmune diseases and disorders. In addition, retrospective analyses suggest that treatment with RCI for RA, SLE, and DM/PM was associated with substantial reductions in the use of corticosteroids, biologics, and nonbiologic DMARDs. Furthermore, initiation of RCI therapy may be associated with subsequent lower healthcare utilization, including decreases in hospitalizations, hospital length of stay, and outpatient visits. The evidence suggests that RCI may improve inflammatory and autoimmune disease control and patient quality of life, particularly in complex patients, and yield healthcare cost savings that demonstrate the medicine's value.

\section{ACKNOWLEDGEMENTS}

Writing support was provided by Marcia L. Reinhart and Judith S. Hurley, and editorial support was provided by Helen Barham and Esther Tazartes of Global Outcomes Group. The 
authors also recognize Tara Nazareth and Winnie Nelson for their critical review of the manuscript. The study, article processing charges, editorial support, and Open Access fee were funded by Mallinckrodt Pharmaceuticals Inc. (Hampton, NJ, USA). All authors had full access to all of the data in this study and take complete responsibility for the integrity of the data and accuracy of the data analysis. All named authors meet the International Committee of Medical Journal Editors (ICMJE) criteria for authorship of this manuscript, take responsibility for the integrity of the work as a whole, and have given final approval for the version to be published.

Disclosures. The authors of this manuscript disclose the following possible financial or personal relationships with commercial entities that may have a direct or indirect interest in the subject matter of this presentation: Michael Philbin was an employee of Mallinckrodt Pharmaceuticals Inc. at the time of the study. He is now an employee of Teva Pharmaceuticals. John Niewoehner is an employee of Mallinckrodt Pharmaceuticals Inc. George J. Wan is an employee of Mallinckrodt Pharmaceuticals Inc.

Compliance with Ethics Guidelines. This article is based on previously conducted studies and does not involve any new studies of human or animal subjects performed by any of the authors.

Data Availability. Data sharing is not applicable to this article as no datasets were generated or analyzed during the current study. This article is based on previously conducted studies and does not involve any new studies of human or animal subjects performed by any of the authors.

Open Access. This article is distributed under the terms of the Creative Commons Attribution-NonCommercial 4.0 International License (http://creativecommons.org/licenses/ by-nc/4.0/), which permits any noncommercial use, distribution, and reproduction in any medium, provided you give appropriate credit to the original author(s) and the source, provide a link to the Creative Commons license, and indicate if changes were made.

\section{REFERENCES}

1. H.P. Acthar Gel (repository corticotropin injection) [prescribing information]. Hazelwood: Mallinckrodt ARD, Inc.; 2015.

2. Berkovich R, Agius MA. Mechanisms of action of ACTH in the management of relapsing forms of multiple sclerosis. Ther Adv Neurol Disord. 2014;7(2):83-96.

3. Gong R. Leveraging melanocortin pathways to treat glomerular diseases. Adv Chronic Kidney Dis. 2014;21(2):134-51.

4. American Academy of Managed Care Pharmacy (AMCP) Dossier, H.P. Acthar ${ }^{\circledR}$ Gel (repository corticotropin injection). St. Louis: Mallinckrodt Pharmaceuticals, Inc.; 2016.

5. Mackay MT, Weiss SK, Adams-Webber T, et al. Practice parameter: medical treatment of infantile spasms: report of the American Academy of Neurology and the Child Neurology Society. Neurology. 2004;62(10):1668-81.

6. Go CY, Mackay MT, Weiss SK, et al. Evidence-based guideline update: medical treatment of infantile spasms. Report of the guideline development subcommittee of the American Academy of Neurology and the Practice Committee of the Child Neurology Society. Neurology. 2012;78(24):1974-80.

7. Pellock JM, Hrachovy R, Shinnar S, et al. Infantile spasms: a U.S. consensus report. Epilepsia. 2010;51(10):2175-89.

8. Shields WD. Infantile spasms: little seizures, BIG consequences. Epilepsy Curr. 2006;6(3):63-9.

9. An S, Nagarajan E, Sánchez Fernández I, et al. Time elapsed from onset of infantile spasms to diagnosis and treatment. Epilepsy Curr. 2015;15(Suppl 1):198-9.

10. Watemberg N. Infantile spasms: treatment challenges. Curr Treat Options Neurol. 2012;14(4):322-31.

11. U.S. Food and Drug Administration. Orphan Drug Designations and Approvals: H.P. Acthar Gel. 2003. https://www.accessdata.fda.gov/scripts/opdlisting/ oopd/. Accessed 16 May 2016. 
12. Baram TZ, Mitchell WG, Tournay A, Snead OC, Hanson RA, Horton EJ. High-dose corticotropin $(\mathrm{ACTH})$ versus prednisone for infantile spasms: a prospective, randomized, blinded study. Pediatrics. 1996;97(3):375-9.

13. Knupp KG, Coryell J, Nickels KC, et al. Response to treatment in a prospective national infantile spasms cohort. Ann Neurol. 2016;79(3):475-84.

14. Hrachovy RA, Frost JD Jr, Glaze DG. High-dose, long-duration versus low-dose, short-duration corticotropin therapy for infantile spasms. J Pediatr. 1994;124(5 Pt 1):803-6.

15. Hrachovy RA, Frost JD Jr, Kellaway P, Zion TE. Double-blind study of ACTH vs prednisone therapy in infantile spasms. J Pediatr. 1983;103(4):641-5.

16. Hrachovy RA, Frost JD Jr, Kellaway P, Zion T. A controlled study of ACTH therapy in infantile spasms. Epilepsia. 1980;21(6):631-6.

17. Love S. Demyelinating diseases. J Clin Pathol. 2006;59(11):1151-9.

18. Rudick RA, Polman CH. Current approaches to the identification and management of breakthrough disease in patients with multiple sclerosis. Lancet Neurol. 2009;8(6):545-59.

19. Gettig J, Cummings JP, Matuszewski KHP. Acthar Gel and cosyntropin review: clinical and financial implications. P T. 2009;34(5):250-7.

20. Rose AS, Kuzma JW, Kurtzke JF, Namerow NS, Sibley WA, Tourtellotte WW. Cooperative study in the evaluation of therapy in multiple sclerosis. ACTH vs. placebo-final report. Neurology. 1970;20(5): $1-59$.

21. Simsarian JP, Saunders C, Smith DM. Five-day regimen of intramuscular or subcutaneous self-administered adrenocorticotropic hormone gel for acute exacerbations of multiple sclerosis: a prospective, randomized, open-label pilot trial. Drug Des Devel Ther. 2011;5:381-9.

22. National Multiple Sclerosis Society. Managing relapses. http://www.nationalmssociety.org/ Treating-MS/Managing-Relapses. Accessed 2 Nov 2016.

23. Cortese I, Chaudhry V, So YT, Cantor F, Cornblath DR, Rae-Grant A. Evidence-based guideline update: plasmapheresis in neurologic disorders. Report of the therapeutics and technology assessment subcommittee of the American Academy of Neurology. Neurology. 2011;76(3):294-300.

24. Keddis MT, Karnath BM. The nephrotic syndrome. Hosp Physician. 2007;38:25-30.
25. Gkrouzman E, Kirou KA, Seshan SV, Chevalier JM. Minimal change disease as a secondary and reversible event of a renal transplant case with systemic lupus erythematosus. Case Rep Nephrol. 2015;2015: 987212.

26. Dember LM. Amyloidosis-associated kidney disease. J Am Soc Nephrol. 2006;17(12):3458-71.

27. Bomback AS, Canetta PA, Beck LH Jr, Ayalon R, Radhakrishnan J, Appel GB. Treatment of resistant glomerular diseases with adrenocorticotropic hormone gel: a prospective trial. Am J Nephrol. 2012;36(1):58-67.

28. Bomback AS, Tumlin JA, Baranski J, et al. Treatment of nephrotic syndrome with adrenocorticotropic hormone (ACTH) gel. Drug Des Devel Ther. 2011;5:147-53.

29. Hogan J, Bomback AS, Mehta K, et al. Treatment of idiopathic FSGS with adrenocorticotropic hormone gel. Clin J Am Soc Nephrol. 2013;8(12):2072-81.

30. Hladunewich MA, Cattran D, Beck LH, et al. A pilot study to determine the dose and effectiveness of adrenocorticotrophic hormone (H.P. Acthar ${ }^{\circledR} \mathrm{Gel}$ ) in nephrotic syndrome due to idiopathic membranous nephropathy. Nephrol Dial Transpl. 2014;29(8):1570-7.

31. Madan A, Mijovic-Das S, Stankovic A, Teehan G, Milward AS, Khastgir A. Acthar gel in the treatment of nephrotic syndrome: a multicenter retrospective case series. BMC Nephrol. 2016;17(1):37.

32. McInnes IB, Schett G. The pathogenesis of rheumatoid arthritis. N Engl J Med. 2011;365(23): 2205-19.

33. Cutolo M, Kitas GD, van Riel PL. Burden of disease in treated rheumatoid arthritis patients: going beyond the joint. Semin Arthritis Rheum. 2014;43(4):479-88.

34. Gaylis N, Needell S, Sagliani J. The effect of adrenocorticotropin gel (HP Acthar Gel) in combination with MTX in newly diagnosed RA patients from a clinical and structural perspective. Ann Rheum Dis. 2015;74(Suppl 2):1066-7.

35. Gillis TM, Crane M, Hinkle C, Wei N. H.P. Acthar Gel (repository corticotropin injection) as adjunctive therapy in patients with rheumatoid arthritis who have failed at least three biologic therapies with different modes of action. Ann Rheum Dis. 2015;74(Suppl 2):1066.

36. Alexanderson $\mathrm{H}$, Lundberg IE. Disease-specific quality indicators, outcome measures and guidelines in polymyositis and dermatomyositis. Clin Exp Rheumatol. 2007;25(6 Suppl 47):153-8. 
37. Gordon PA, Winer JB, Hoogendijk JE, Choy EH. Immunosuppressant and immunomodulatory treatment for dermatomyositis and polymyositis. Cochrane Database Syst Rev. 2012;8:Cd003643.

38. Dimachkie MM, Barohn RJ. Idiopathic inflammatory myopathies. Front Neurol Neurosci. 2009;26:126-46.

39. Levine T. Treating refractory dermatomyositis or polymyositis with adrenocorticotropic hormone gel: a retrospective case series. Drug Des Dev Ther. 2012;6:133-9.

40. Aggarwal RM, Marder G, Loganathan P, et al. Efficacy and safety of adrenocorticotropic hormone gel (Acthar $\mathrm{Gel}^{\circledR}$ ) in refractory dermatomyositis or polymyositis. Abstract presented at American College of Rheumatology/Association of Rheumatology Health Professionals Annual Meeting [abstract no. 2363]; November 6-11, 2015; San Francisco.

41. Davis LS, Hutcheson J, Mohan C. The role of cytokines in the pathogenesis and treatment of systemic lupus erythematosus. J Interferon Cytokine Res. 2011;31(10):781-9.

42. Marian V, Anolik JH. Treatment targets in systemic lupus erythematosus: biology and clinical perspective. Arthritis Res Ther. 2012;14(Suppl 4):S3.

43. Guidelines for referral and management of systemic lupus erythematosus in adults. American College of Rheumatology Ad Hoc Committee on Systemic Lupus Erythematosus Guidelines. Arthritis Rheum. 1999;42(9):1785-1796.

44. Fiechtner JJ, Montroy T. Treatment of moderately to severely active systemic lupus erythematosus with adrenocorticotropic hormone: a single-site, open-label trial. Lupus. 2014;23(9):905-12.

45. Furie R, Mitrane M, Zhao E, Das M, Li D, Becker PM. Efficacy and tolerability of repository corticotropin injection in patients with persistently active SLE: results of a phase 4 , randomised, controlled pilot study. Lupus Sci Med. 2016;3(1):e000180.

46. Iannuzzi MC, Rybicki BA, Teirstein AS. Sarcoidosis. N Engl J Med. 2007;357(21):2153-65.

47. Baughman RP, Culver DA, Judson MA. A concise review of pulmonary sarcoidosis. Am J Respir Crit Care Med. 2011;183(5):573-81.

48. Foundation for Sarcoidosis Research. Sarcoidosis treatment guidelines. 2013. http://www.stopsar coidosis.org/wp-content/uploads/2013/03/FSR-Physi cians-Protocol1.pdf. Accessed 16 May 2016.
49. Beegle SH, Barba K, Gobunsuy R, Judson MA. Current and emerging pharmacological treatments for sarcoidosis: a review. Drug Des Dev Ther. 2013;7:325-38.

50. O'Regan A, Berman JS. Sarcoidosis. Ann Intern Med. 2012;156(9):ITC5-1-15.

51. Salomon A, Appel B, Collins SF, Herschfus JA, Segal MS. Sarcoidosis: pulmonary and skin studies before and after ACTH and cortisone therapy. Dis Chest. 1956;29(3):277-91.

52. Baughman RP, Barney JB, O'Hare L, Lower EE. A retrospective pilot study examining the use of Acthar gel in sarcoidosis patients. Respir Med. 2016;110:66-72.

53. Gold LS, Schepman PB, Wang WJ, et al. Healthcare costs and resource utilization in patients with infantile spasms treated with H.P. Acthar Gel ${ }^{\circledR}$. Adv Ther. 2016;33(8):1293-304.

54. Gold LS, Suh K, Schepman PB, Damal K, Hansen RN. Healthcare costs and resource utilization in patients with multiple sclerosis relapses treated with H.P. Acthar $\mathrm{Gel}^{\circledR}$. Adv Ther. 2016;33(8): 1279-92.

55. Wu B, Deshpande G, Popelar B, Wan G, Philbin M. Real-world treatment patterns and demographic, clinical and economic characteristics of rheumatoid arthritis patients initiating repository corticotropin injection therapy. American Society of Health-System Pharmacists Summer Meeting; Jun 11-15, 2016; Baltimore.

56. Knight T, Bond C, Popelar B, Wang L, Philbin M. Medical resource utilization in dermatomyositis/ polymyositis patients treated with repository corticotropin injection, intravenous immunoglobulin, and/or rituximab. American Society of Health-System Pharmacists Summer Meeting; Jun 11-15, 2016; Baltimore.

57. Wu B, Deshpande G, Tunceli O, et al. Real-world treatment patterns and demographic, clinical, and economic characteristics of systemic lupus erythematosus (SLE) patients initiating repository corticotropin injection therapy. AMCP Managed Care \& Specialty Pharmacy Annual Meeting 2016; April 19-22, 2016; San Francisco.

58. Myung G, Nelson WW, McMahon MA. Effects of repository corticotropin injection on medication use in patients with rheumatologic conditions: a claims data study. American College of Rheumatology Annual Meeting; May 15-19, 2016; Washington. 